

\title{
EVALUACIÓN DE LA CORROSIÓN POR PICADURA EN ALEACIONES DE ALUMINIO
}

\author{
Luis Garita Arce \\ Lino Rivolta Carvallo \\ Mario Vega León
}

\begin{abstract}
Resumen
El objetivo de este proyecto fue evaluar la corrosión por picadura en recipientes de cocina, fabricados a partir de aleaciones de aluminio; mediante la caracterización microscópica (metalografía y microscopía electrónica de barrido) de las mismas y su comportamiento electroquímico en un medio en específico.

Las muestras utilizadas fueron las aleaciones: 1050, 1050 baja en cobre, 1200 y una aleación 1050 pero que ya había sido usado por parte del usuario final, la cual había presentado picaduras.

Entre los resultados más importantes se tiene que los distintos materiales utilizados no presentan diferencias en cuanto a susceptibilidad de picadura en igualdad de condiciones, según norma ASTM G100-89 Standard Test Method for Conducting Cyclic Galvanostaircase Polarization.
\end{abstract}

Palabras clave: Corrosión por picadura, microscopía, ensayos electroquímicos, aleación de aluminio.

\begin{abstract}
The main objective of this investigation was to study pitting corrosion on aluminum pots by microscopic characterization and their electrochemical behavior in a specific job solution. The type of materials employed were the following alloys: 1050, 1050 low copper content, 1200, and lastly the particular alloy material AA1050 was used, although it had already been used by an end user and had showed pitting.Among the most substantial results, one can state that the different materials analyzed do not indicate differences as for the pitting susceptibilities in equal conditions; all of this was done according to norm ASTM G100-89 Standard Test Method for Conducting Cyclic Galvanostaircase Polarization.
\end{abstract}

Keywords: Pitting corrosion, microscopy, aluminum alloy.

Recibido: 11 de marzo del 2011 • Aprobado: 11 de junio del 2013

\section{INTRODUCCIÓN}

Diferentes tipos de metales son utilizados en una gran cantidad de aplicaciones, tanto en infraestructura, uso industrial o doméstico, dentro de los que pueden mencionarse tanques, reactores, tuberías, bombas, edificaciones y hasta como utensilios de cocina, de allí la importancia del estudio de los diferentes tipos de corrosión tales como la generalizada, localizada, entre otras.

En una planta procesadora se producen discos de aluminio que luego son comercializados para la fabricación de recipientes y utensilios de cocina mediante los procesos de repujado y embutido, en ocasiones estos utensilios sufren corrosión localizada del tipo picadura.

El presente proyecto tiene como objetivo evaluar la corrosión por picadura en recipientes de cocina, fabricados a partir de aleaciones de aluminio, para ello se llevará a cabo una caracterización de las aleaciones utilizadas mediante microscopía óptica y microscopía electrónica; además, se determinará el potencial de protección para las aleaciones, utilizando un potenciostato y la técnica electroquímica de Polarización Cíclica Galvanostática Escalonada (PCGE). 


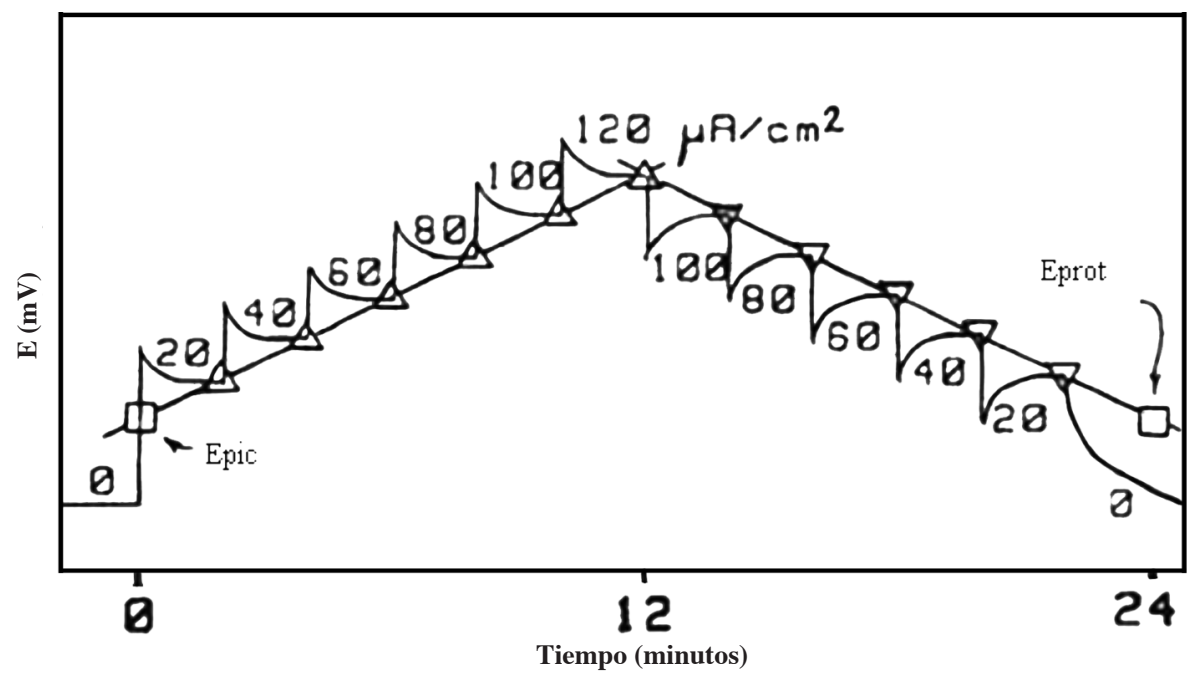

Figura 1. Curva PCGE esquemática para un material susceptible a desarrollar corrosión por picadura.

Fuente: Rivolta, 2008.

\subsection{Corrosión por picadura}

Este tipo de corrosión localizada es caracterizada por la formación de cavidades irregulares en la superficie del metal. El diámetro y profundidad de la cavidad dependen de varios parámetros relacionados con el metal, el medio y las condiciones de servicio:

"La corrosión por picaduras ocurre cuando el metal se pone en contacto permanente o intermitente con medios acuosos: agua, agua de mar, agua de lluvia o humedad. La experiencia demuestra que cuando se da corrosión por picadura, se desarrollará siempre durante las primeras semanas de exposición.”(Vargel, 2004)

\subsection{Polarización Cíclica Galvanostática Escalonada (PCGE)}

Según Rivolta (2008), los potenciales de picadura y de protección son parámetros que definen el comportamiento electroquímico de un material frente a la pasivación. Su obtención puede realizarse mediante diferentes métodos electroquímicos. Por ejemplo, la polarización galvanostática cuasiestacionaria mediante el cambio de corriente en escalones predeterminados a tiempos dados, denominada también como polarización cíclica galvanostática escalonada (PCGE). Con esta técnica, las variaciones en el potencial de electrodo son registrados al ir cambiando escalones de densidad de corriente a tiempos predeterminados. Los cambios de estos escalones de densidad de corriente están acotados por un mínimo en 0 y un máximo establecido, constituyendo la rama ascendente de la curva, para luego finalizar el ensayo con la rama descendente desde el máximo valor establecido hasta cero. La determinación de los potenciales de picadura y de protección del material se obtiene al extrapolar a una recta cada uno de los potenciales cuasiestacionarios de la rama de ascenso para los potenciales de picadura, y de la rama de descenso para los potenciales de protección.

En la Figura 1 se puede observar esquemáticamente una representación de este ensayo:

"En la figura anterior se observa una curva PCGE realizada a un material metálico capaz de establecer una película pasiva y desarrollar corrosión localizada por picadura durante el ensayo. Este ensayo de PCGE fue realizado con intervalos de corriente de $20 \mu \mathrm{A} / \mathrm{cm}^{2}$ cada 2 minutos, desde $0 \mu \mathrm{A} / \mathrm{cm}^{2}$ hasta $120 \mu \mathrm{A} / \mathrm{cm}^{2}$, constituyendo la rama en ascenso, y luego de manera descendente hasta alcanzar de nuevo 
$0 \mu \mathrm{A} / \mathrm{cm}^{2}$. La extrapolación de los potenciales cuasiestacionarios, representados por un triangulo, permite la determinación de los potenciales de picadura $\left(\mathrm{E}_{\mathrm{pic}} \mathrm{y}\right.$ de protección $\left(\mathrm{E}_{\text {prot }}\right)$ ". (Rivolta, 2008)

Según Hirozawa (1986), las ventajas de ésta técnica son la exactitud y rapidez, debido a su reproducibilidad en la obtención del potencial de protección y la eliminación del efecto del tiempo de inducción de la picadura, además del lazo de histéresis de las curvas cíclicas potenciodinámicas. Por otra parte, todo el ataque localizado que pueda desarrollarse sobre el material estará ubicado en las zonas más susceptibles.

\section{METODOLOGÍA EXPERIMENTAL}

La fase experimental se divide en dos partes: la primera consiste en la caracterización del material, donde se realiza un análisis de composición química de la aleación en cuestión mediante espectrometría de emisión óptica, análisis metalográfico con ayuda de microscopía óptica, y por último, un análisis elemental mediante dispersión de rayos X (EDS); la segunda parte corresponde a los ensayos electroquímicos en la cual se determina el potencial de protección mediante la técnica de polarización cíclica galvanostática escalonada.

Con respecto a la primera parte, para el análisis metalográfico se hace un montaje de aproximadamente un centímetro cuadrado de la aleación de interés en una resina epóxica mediante una prensa de montaje automática; luego se lleva a cabo el pulido con un pulidor automático, proceso en el que se lija secuencialmente con papel abrasivo No. 120, 240, 320, 400 y 600 durante 30 $\mathrm{s}$ con una fuerza de $89 \mathrm{~N}$ y $15.71 \mathrm{rad} / \mathrm{s}$ (150 rpm); luego se pule a $15.71 \mathrm{rad} / \mathrm{s}(150 \mathrm{rpm})$ y una fuerza de $80 \mathrm{~N}$ con pasta de diamante de $15 \mu \mathrm{m}$ por 90 $\mathrm{s}$, después con pasta de $6 \mu \mathrm{m}$, de $3 \mu \mathrm{m}$ y de $1 \mu \mathrm{m}$ por $150 \mathrm{~s}$ cada vez. Para finalizar de pulir se aplica sílica coloidal con una fuerza de $67 \mathrm{~N}, 15.71 \mathrm{rad} / \mathrm{s}$ (150 rpm) y un tiempo de 5 minutos, lo que da un acabado espejo a las muestras.

Una vez pulidas las muestras se les efectúa una inspección metalográfica, luego se les realiza un ataque en un electropulidor mediante una solución electrolítica y se someten de nuevo a inspección metalográfica.

Las pruebas electroquímicas se realizan mediante la técnica polarización cíclica galvanostática escalonada, según la norma ASTM G100-89 Standard Test Method for Conducting Cyclic Galvanostaircase Polarization.

\section{RESULTADOS Y DISCUSIÓN}

\subsection{Análisis de composición química}

En el Cuadro 1 se presentan las composiciones teóricas de las aleaciones en estudio según American Society for Metals (1992), donde el porcentaje de Al es el mínimo especificado y el de los aleantes es el valor máximo especificado; además, para la aleación 1200, el Si y el Fe deben sumar como máximo $1 \%$. En el Cuadro 2 se presenta el análisis de composición química realizado a los distintos materiales en estudio; teniendo en cuenta que A es la aleación 1050, B es la aleación 1050 baja en $\mathrm{Cu}, \mathrm{C}$ es la aleación 1200 y D es la aleación 1050 que presentó picaduras luego de que se puso en servicio. Al comparar los valores teóricos con los reales, se observa que las distintas aleaciones no sobrepasan los valores máximos de aleantes; la muestra $\mathrm{C}$ contiene su valor mínimo de aluminio, mientras que las muestras $\mathrm{A}$ y $\mathrm{D}$, contienen un valor de $\mathrm{Al}$ inferior al mínimo de la norma, situación que puede deberse a que el análisis se realizó a una muestra de lámina y no a una normalizada tomada del aluminio líquido del canal de colado.

\subsection{Microscopía óptica}

La Figura 2 presenta las metalografías de las distintas aleaciones en estudio, vistas a 100X sin atacar. Como se puede observar, las cuatro muestras se comportan de forma similar, de acuerdo con las aleaciones de la serie 1000, sólo en la Figura 2B (correspondiente a la aleación 1050 baja en $\mathrm{Cu}$ ) hay más presencia de puntos negros. Estos puntos se evalúan mas adelante 
Cuadro 1. Composición elemental teórico

\begin{tabular}{ccc}
\hline Elementos & $\begin{array}{c}\text { Composición Aleación 1050\% } \\
(\mathbf{m} / \mathbf{m})\end{array}$ & $\begin{array}{c}\text { Composición Aleación 1200\% } \\
(\mathbf{m} / \mathbf{m})\end{array}$ \\
\hline $\mathrm{Al}_{\min }$ & 99,50 & 99 \\
$\mathrm{Si}$ & 0,25 & $1,00 \mathrm{Si+Fe}$ \\
$\mathrm{Fe}$ & 0,40 & -- \\
$\mathrm{Cu}$ & 0,05 & 0,05 \\
$\mathrm{Mn}$ & 0,05 & 0,05 \\
$\mathrm{Mg}$ & 0,05 & -- \\
$\mathrm{Zn}$ & 0,05 & 0,10 \\
$\mathrm{~V}$ & 0,05 & -- \\
$\mathrm{Ti}$ & 0,03 & 0,05 \\
\hline
\end{tabular}

Fuente: American Society for Metals, 1992.

Cuadro 2. Composición elemental para las diferentes aleaciones de aluminio utilizadas

\section{Elementos}

$\begin{array}{ccccc} & \mathbf{A} & \mathbf{B} & \mathbf{C} & \mathbf{D} \\ \mathrm{Al}_{\text {min }} & 99,46 & 99,55 & 99,4 & 99,490 \\ \mathrm{Si} & 0,13 & 0,11 & 0,15 & 0,140 \\ \mathrm{Fe} & 0,30 & 0,42 & 0,310 \\ \mathrm{Cu} & 0,34 & 0,015 & 0,003 & 0,031 \\ \mathrm{Mn} & 0,031 & 0,0029 & 0,051 & 0,002 \\ \mathrm{Mg} & 0,011 & 0,0023 & 0,0019 & 0,002 \\ \mathrm{Zn} & 0,0006 & 0,002 & 0,0016 & 0,003 \\ \mathrm{~V} & 0,0027 & 0,009 & 0,009 & 0,008 \\ \mathrm{Ti} & 0,009 & 0,017 & 0,002 & 0,017\end{array}$


con un análisis de rayos $\mathrm{X}$ por dispersión (EDS) mediante un análisis de composición elemental en dichas regiones de interés.

En la Figura 3 se observan las mismas muestras a 100X pero atacadas electrolíticamente, todas presentan un patrón similar; sin embargo, la muestra C (aleación 1200) presenta una microestructura diferente, es decir el tamaño de grano es más pequeño. Esta situación puede considerarse normal, ya que dicha aleación proviene de un proceso de laminación en caliente. Por otra parte, en la muestra D (material que presentó picadura), se puede apreciar dos picaduras presentes en el material.

La Figura 4 muestra una picadura presente en el material observado en un estereomicroscopio. En ésta se observa una formación blanquecina, la cual es un producto de corrosión llamado alúmina, típico de corrosión en picaduras sobre aluminio, tal como comenta Vargel (2004).

\subsection{Microscopía electrónica de barrido}

\section{Inspección Visual}

La Figura 5 presenta las muestras atacadas electrolíticamente de las diferentes aleaciones, vistas al microscopio electrónico de barrido (MEB). En todas se encuentra una matriz metálica muy homogénea, este comportamiento es esperado debido a la alta pureza de las mismas. En ninguna de ellas se observan presencia de otras fases; sin embargo, en todas las muestras se encontraron puntos blancos distribuidos uniformemente en todo el material, tanto en los bordes de grano como en el interior de los mismos, este efecto es más notorio en la muestra $\mathrm{C}$, que es analizada, luego, mediante Análisis elemental por dispersión de rayos X (EDS) como se observa en la Figura 8.

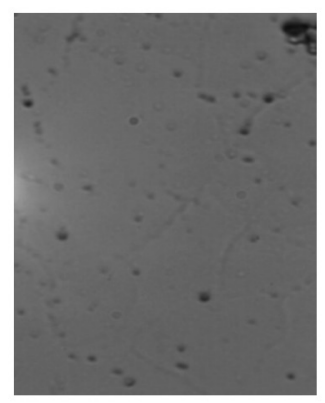

A

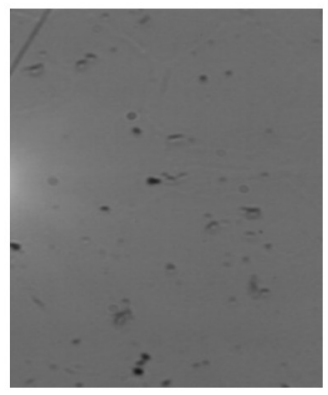

$\mathrm{C}$

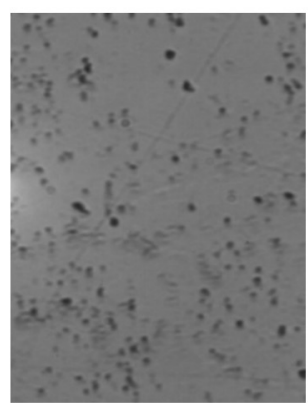

B

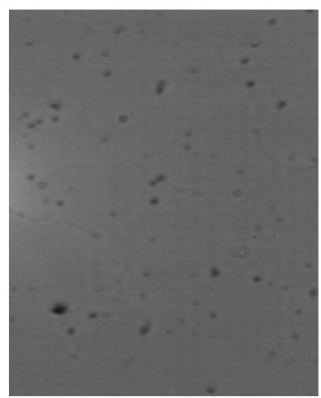

$\mathrm{D}$

Figura 2. Metalografías de los diferentes materiales vistas a 100X sin ataque, donde se observan diferentes densidades de segundas fases (indicados por los puntos negros). A) Aleación 1050. B) Aleación 1050 baja en Cu. C) Aleación 1200.

D) Aleación 1050 que presentó picadura.

Fuente: Elaboración propia, 2012. 


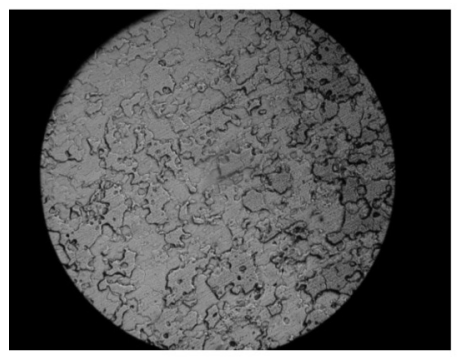

A

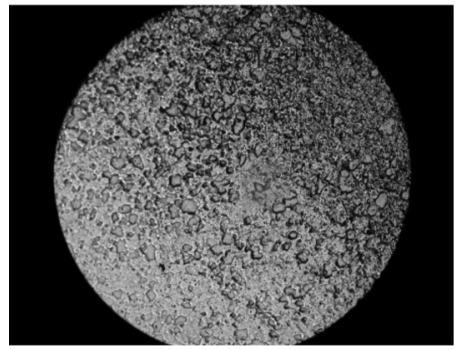

$\mathrm{C}$

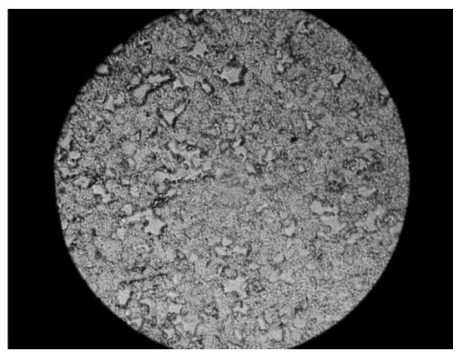

B

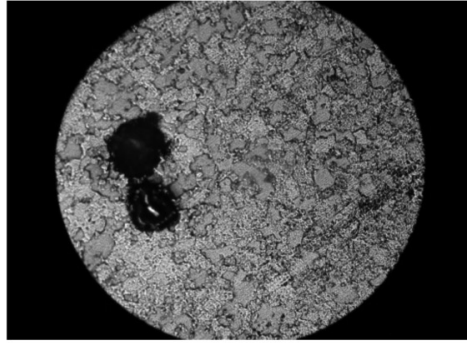

$\mathrm{D}$

Figura 3. Metalografías de los diferentes materiales vistas a 100X atacadas electrolíticamente. A) Aleación 1050. B) Aleación 1050 baja en contenido de Cobre. C) Aleación 1200. D) Aleación 1050 con picadura (manchas negras a la izquierda de la fotografía).

Fuente: Elaboración propia, 2012.

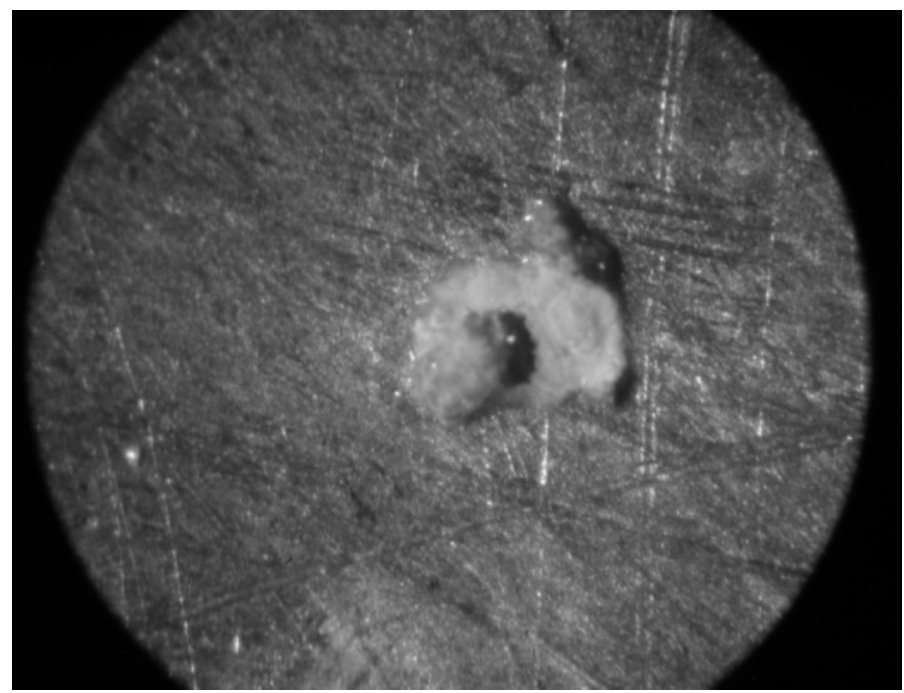

Figura 4. Picadura encontrada en aleación 1050 bajo condiciones de uso a 20X (Abultamiento blanquecino en el centro de la fotografía)

Fuente: Elaboración propia, 2012. 


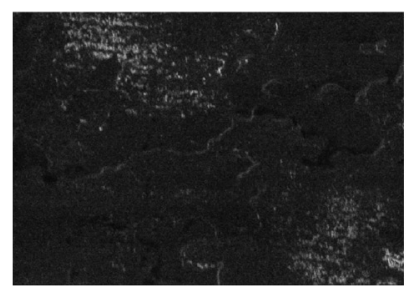

A

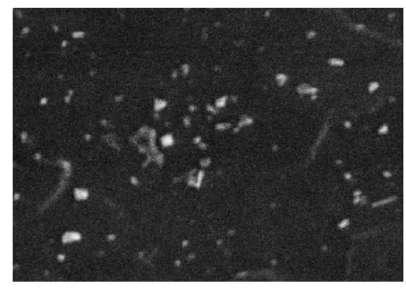

$\mathrm{C}$

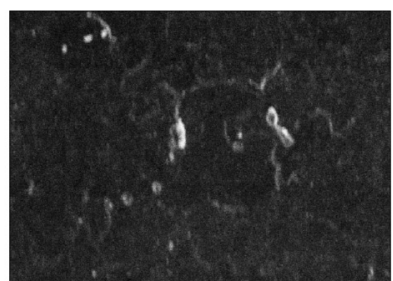

$\mathrm{B}$

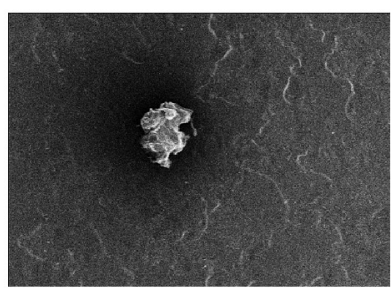

$\mathrm{D}$

Figura 5. Microscopía electrónica de aleaciones de Al con ataque electrolítico. A) Aleación 1050 a 220X. B) Aleación 1050 baja en Cu a 350X. C) Aleación 1200 a 900X. D) Aleación 1050 que presentó picadura a 170X (mancha blanca en el centro de la fotografía).

Fuente: Elaboración propia, 2012.

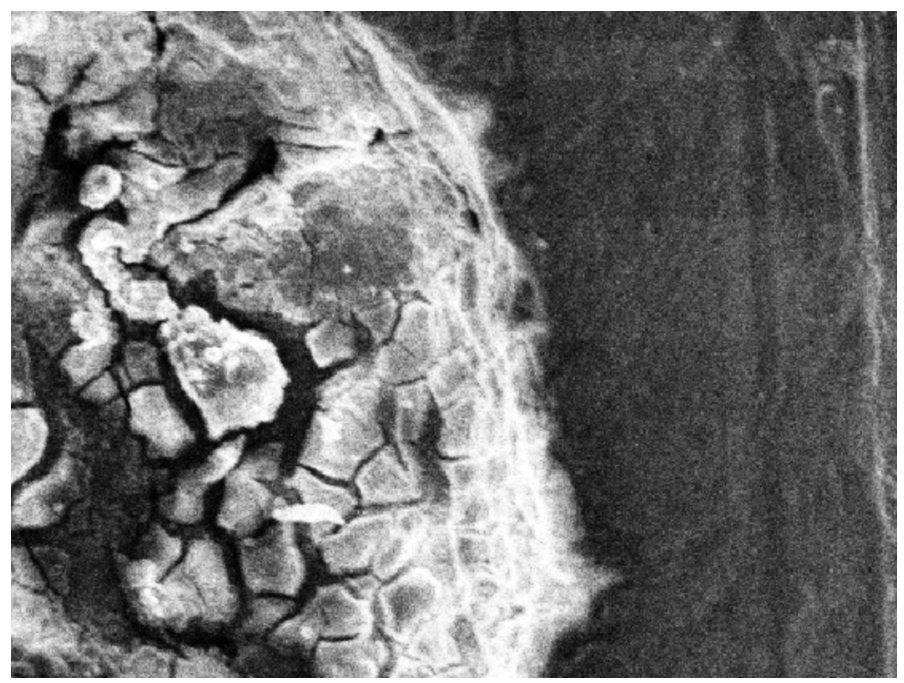

Figura 6. Producto de la corrosión por picadura presente en la aleación 1050 en condiciones de uso a $330 \mathrm{X}$.

Fuente: Elaboración propia, 2012. 
La muestra D de la Figura 5 captura una picadura ocurrida en condiciones de uso, donde se observa una gran mancha blanca. Sobre esta picadura se realiza un acercamiento, como se muestra en la Figura 6 para observar con mayor detalle su morfología.

\section{Análisis elemental por dispersión de rayos $X(E D S)$}

Se realizaron análisis EDS a lo largo de la matriz metálica y en zonas con presencia de picaduras o productos de corrosión, para varias muestras de interés.

La Figura 7 muestra el análisis efectuado a la aleación 1050, éste consiste en realizar un barrido de la composición elemental a través de una línea recta, trazada por las regiones de interés. Como se puede apreciar, en una parte de la línea de análisis existe un pico que corresponde a una alta concentración de silicio, lo cual es típico en estas aleaciones, ya que junto con el hierro es uno de los principales aleantes. De acuerdo con American Society for Metals (1998) estos dos, a su vez, pueden formar segundas fases de carácter catódico favoreciendo a la generación de picaduras

$\mathrm{Al}$ realizar un análisis similar en la aleación 1200 , se traza el vector de análisis de manera que atraviese, además de la matriz metálica, los puntos blancos (ver Figura 8). En las manchas blancas tanto pequeñas como grandes, se encuentra un incremento en la cantidad de cloruros (línea celeste) mientras que la cantidad de oxígeno (línea azul) aumenta considerablemente en las más grandes; esto indica que las manchas pequeñas pueden ser la etapa de iniciación de corrosión por picadura mediante la adsorción del cloruro, seguido por el rompimiento de la capa pasiva, para luego dar paso a la etapa de propagación (manchas blancas grandes), donde se produce hidróxido de aluminio $\mathrm{Al}(\mathrm{OH})_{3} \mathrm{y}$, por ende, una mayor presencia de oxígeno, esto concuerda con el mecanismo propuesto en Vargel (2004).
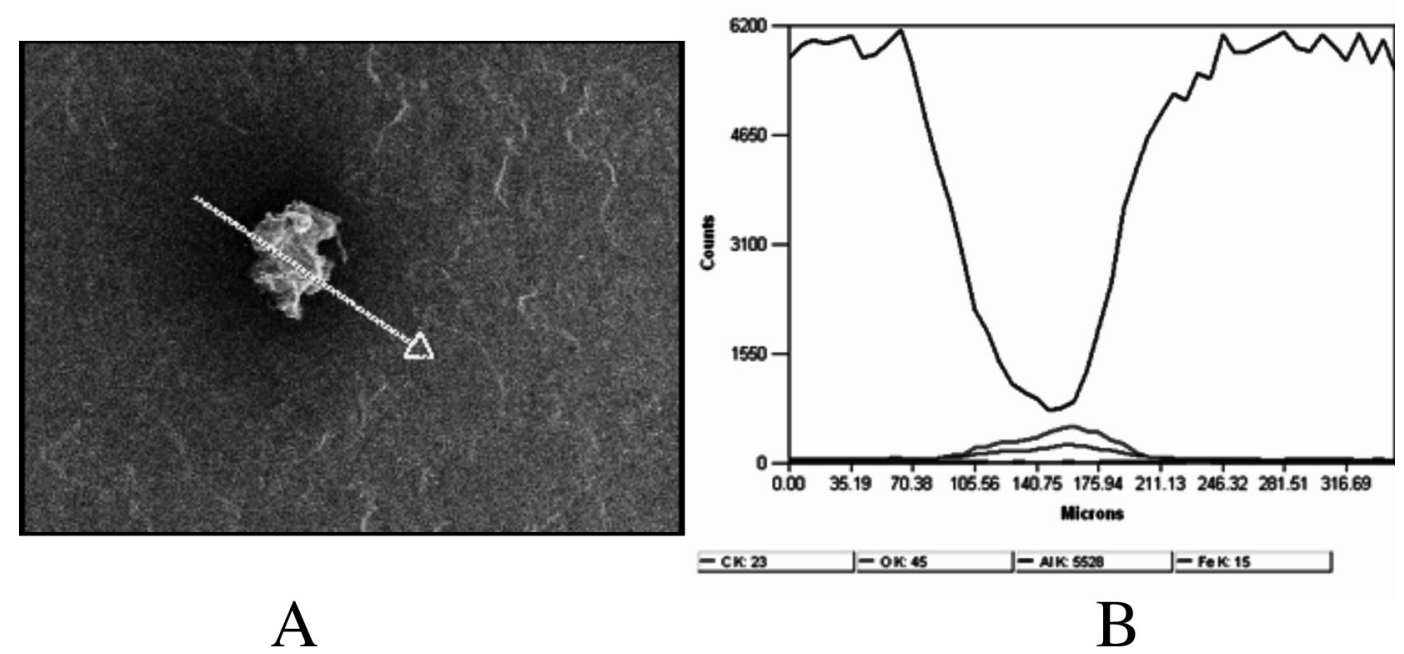

B

Figura 7. Análisis EDS de la aleación 1050 a 100X.

Fuente: Elaboración propia, 2012. 


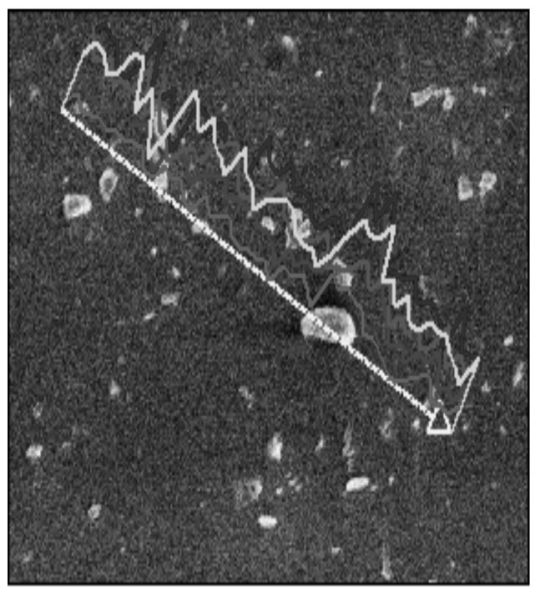

A

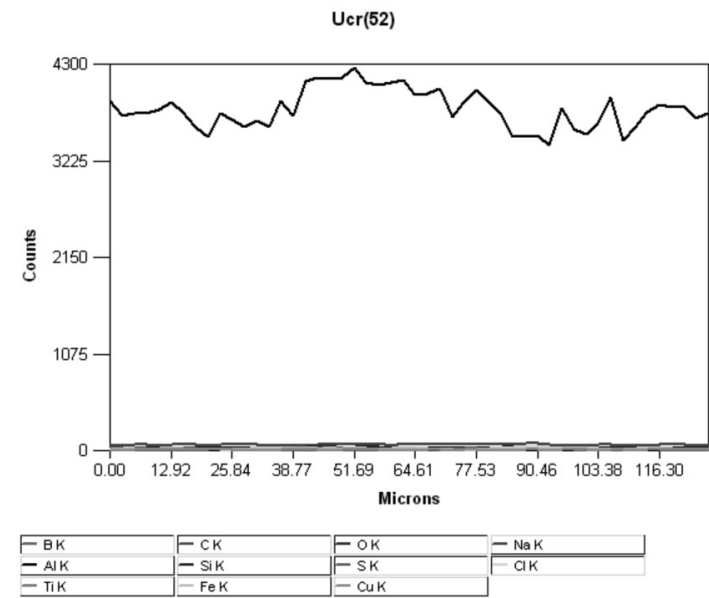

B

Figura 8. Análisis EDS de la aleación 1200 a 900X

Fuente: Elaboración propia, 2012.

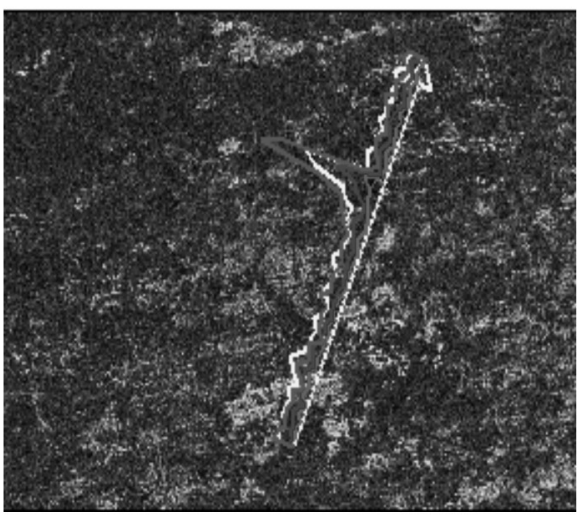

A

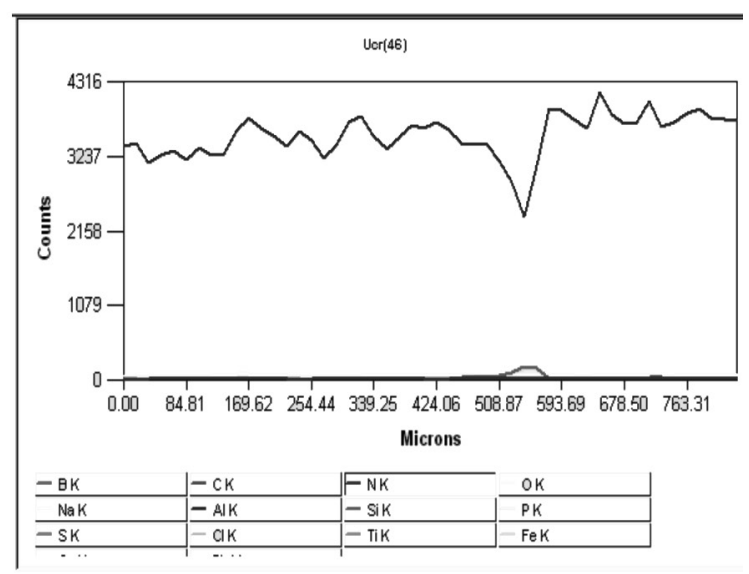

B

Figura 9. Análisis EDS de la aleación 1050 con picaduras a 170X.

Fuente: Elaboración propia, 2012. 


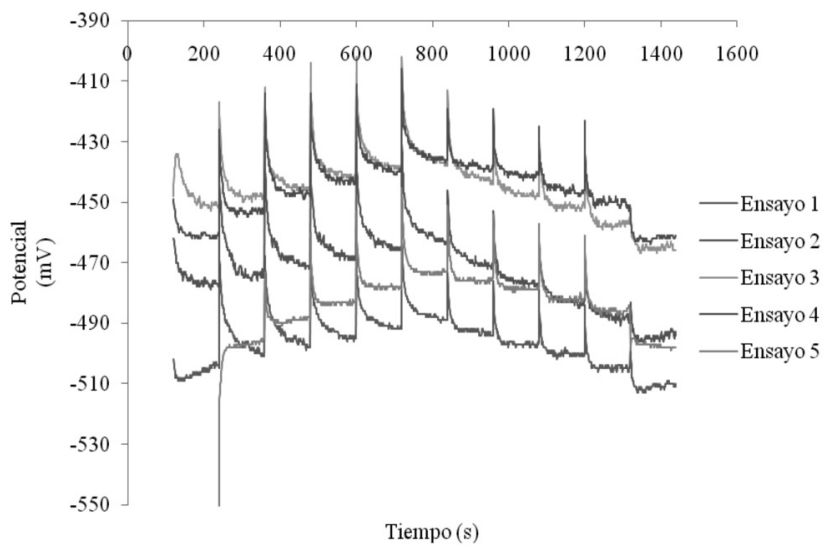

Figura 10. Polarización cíclica galvanostática escalonada para la aleación de aluminio 1050

Fuente: Elaboración propia, 2012.

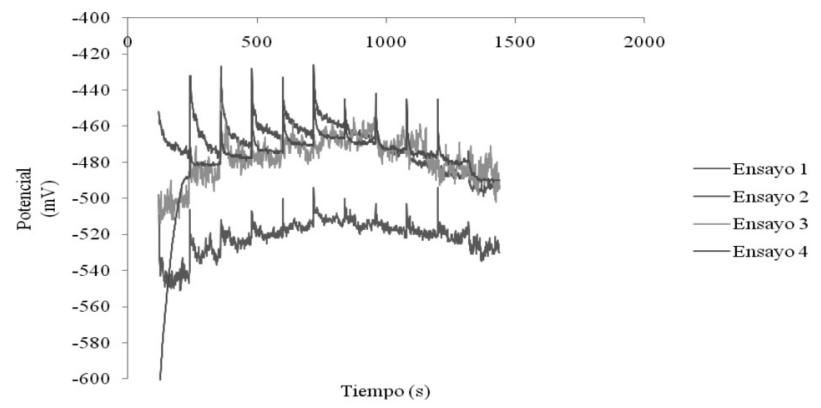

Figura 11. Polarización cíclica galvanostática escalonada para la aleación de aluminio 1050 con contenidos bajos de cobre.

Fuente: Elaboración propia, 2012.

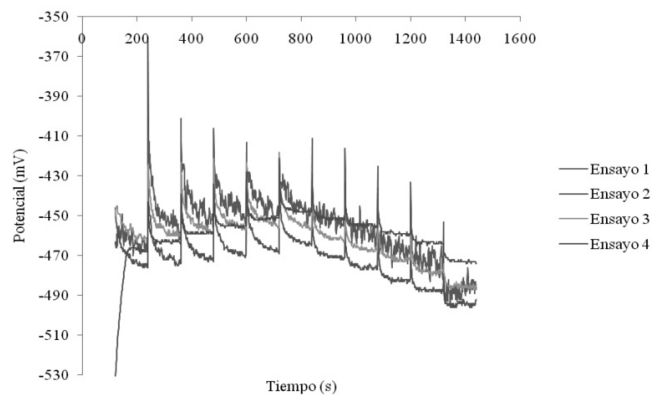

Figura 12. Polarización cíclica galvanostática escalonada para la aleación de aluminio 1200.

Fuente: Elaboración propia, 2012. 


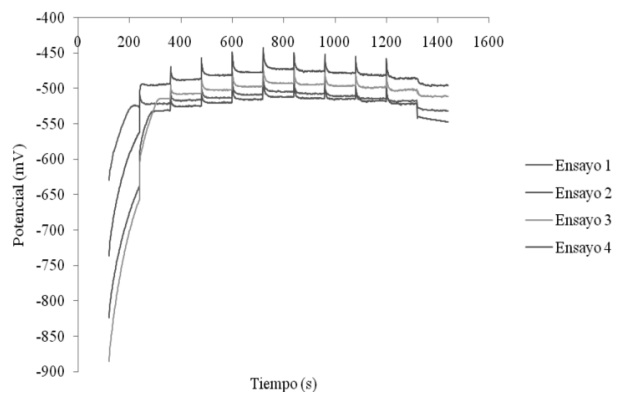

Figura 13. Polarización cíclica galvanostática escalonada para la aleación de aluminio 1050 que presentó picaduras .

Fuente: Elaboración propia, 2012.

Por último, al realizar un análisis elemental a la aleación 1050 con picadura, a través de la línea trazada (ver Figura 9), se puede apreciar que al pasar por la mancha blanca disminuye sustancialmente el aluminio aumentando la cantidad de carbono y oxígeno. Este comportamiento es indicativo de ser una picadura donde existe residuos de material orgánico, además el oxígeno es debido a la producción de alúmina $\mathrm{Al}(\mathrm{OH})_{3}$.

\subsection{Análisis electroquímico}

La técnica de polarización cíclica galvanostática escalonada (PCGE), utilizada para el análisis electroquímico, se realizó siguiendo las pautas de la norma ASTM G10089 Standard Test Method for Conducting Cyclic Galvanostaircase Polarization.

Como se puede ver desde la Figura 10 hasta la Figura 13, los cuatro materiales estudiados tienen un comportamiento típico ya que las curvas contienen una sección ascendente escalonada, correspondiente a la polarización, y luego otra rama descendente escalonada, correspondiente a la despolarización; además, no presenta distorsiones muy pronunciadas, efecto que puede ser atribuido a la homogeneidad de la matriz metálica, como se mostró anteriormente en los análisis de microscopía electrónica de barrido.
La aleación 1050 que había presentado picadura (Figura 13), muestra un comportamiento particular, ya que en su primer escalón de polarización no se obtiene una estabilización del potencial, lo cual implica que con una corriente anódica no presenta el salto de potencial seguido por su estabilización; sin embargo, el escalón típico en estas pruebas se logra a partir del segundo escalón de polarización, pudiéndose aplicar la técnica de ensayo.

El Cuadro 3 muestra los valores del potencial de protección $\left(\mathrm{E}_{\text {prot }}\right)$ para los distintos materiales, según American Society Testing Materials (2004), la aleación que presente los valores más negativos será más sensible a la picadura.

Dado que se efectuaron varias corridas para evaluar Eprot de cada uno de los materiales, se procede a realizar un análisis de varianza con un nivel de significancia del $5 \%$ para determinar si existe diferencia entre las medias de los cuatro tipos de materiales. Para el desarrollo de este análisis, se plantea como hipótesis nula, que no existe diferencia entre las medias:

$$
\mathrm{H} 0: \mu 1=\mu 2=\mu 3=\mu 4
$$

Al realizar el análisis (ver Cuadro 4) se determina que no hay suficiente evidencia estadística para rechazar la hipótesis nula; por tanto, a pesar de las diferencias particulares detectadas en algunas de las aleaciones, tal como 
Cuadro 3. Potenciales de protección para diferentes aleaciones

\begin{tabular}{|c|c|c|c|c|}
\hline Ensayos & $\mathbf{A} \mathbf{E}_{\text {prot }}(\mathbf{m V})$ & B $E_{\text {prot }}(\mathbf{m V})$ & $C E_{\text {prot }}(\mathbf{m V})$ & $D E_{\text {prot }}(\mathrm{mV})$ \\
\hline 1 & $-509,31$ & $-525,62$ & $-486,28$ & $-526,7$ \\
\hline 2 & $-494,13$ & $-494,08$ & $-493,05$ & $-535,97$ \\
\hline 3 & $-463,95$ & $-493,16$ & $-484,02$ & $-506,90$ \\
\hline 4 & $-457,52$ & $-486,51$ & $-470,85$ & $-492,51$ \\
\hline 5 & $-492,93$ & & & $-526,7$ \\
\hline
\end{tabular}

Fuente: Elaboración propia, 2012.

Cuadro 4. Análisis de varianza para el potencial de protección de los distintos materiales

\begin{tabular}{|c|c|c|c|c|c|}
\hline Material & Repeticiones & $\underset{(\mathrm{mV})}{\text { Promedio }} \mathbf{E}_{\text {prot }}$ & Varianza & $\mathbf{F}$ & F Crítico \\
\hline & & - & 481,3 & & \\
\hline \multirow[t]{3}{*}{ A } & 5 & & & & \\
\hline & & 483,57 & 0 & & \\
\hline & & - & 306,7 & & \\
\hline \multirow[t]{3}{*}{ B } & 4 & & & & \\
\hline & & 499,84 & 0 & 3,013 & 3,411 \\
\hline & & - & & & \\
\hline \multirow[t]{3}{*}{$\mathrm{C}$} & 4 & & 86,40 & & \\
\hline & & 483,55 & & & \\
\hline & & - & 382,3 & & \\
\hline \multirow[t]{2}{*}{$\mathrm{D}$} & 4 & & & & \\
\hline & & 515,52 & 2 & & \\
\hline
\end{tabular}

Fuente: Elaboración propia, 2012. 
la microestructura de grano más pequeño de la aleación 1200 (ver Figura 3) o bien una menor cantidad de cobre en la aleación 1050 (ver Cuadro 2), los cuatro materiales se comportan igual electroquímicamente, es decir, todos presentan la misma susceptibilidad a la picadura.

\section{CONCLUSIONES}

- De acuerdo con los resultados del análisis elemental de rayos $\mathrm{X}$ por dispersión (EDS) en la aleación 1050 se encuentran compuestos de silicio, los cuales pueden actuar como cátodos en la formación de la picadura.

- Según el análisis EDS, se encontró presencia de cloruros en la aleación 1200 los cuales pueden participar en el rompimiento de la capa pasiva y dar paso a la formación de la picadura.

- De acuerdo con el análisis de potencial de protección $\left(\mathrm{E}_{\text {prot }}\right)$, según la norma ASTM G100-89, los diferentes tipos de materiales utilizados en igualdad de condiciones, presentan comportamientos con similitud electroquímica, teniendo estadísticamente promedios de $\mathrm{E}_{\text {prot }}$ iguales y por ende los diferentes materiales tienen la misma susceptibilidad a la corrosión por picadura.

\section{AGRADECIMIENTOS}

Se agradece de forma especial el apoyo brindado por parte del Instituto Costarricense de Electricidad (ICE) para el desarrollo de este proyecto.

\section{REFERENCIAS BIBLIOGRAFICAS}

American Society for Metals (ASM). (1992). Corrosion, 13. Estados Unidos: McGraw-Hill.

American Society for Metals (ASM). (1998). Metals Handbook. EEUU: ASM Handbook Committee.

American Society Testing Materials (ASTM). (2004). Standard Test Method for Conducting Cyclic Galvanostaircase Polarization. Annual
Book of Standards. Estados Unidos: McGrawHill.

Hirozawa, S. T. (1986). Corrosion monitoring by Galvanostaircase Polarization. (R. Baboin, Ed.) Electrochemical Techniques for Corrosion Engineering.

Rivolta, L. (2008). Efecto Del Vanadio Sobre la Temperatura Crítica de Picado. Caracas, Venezuela.

Vargel, C. (2004). Corrosion of Aluminium. Estados Unidos: Elsevier.

\section{Literatura Consultada}

Ávila, \& Genescà. (1996). Más allá del Herrumbre (Vol. 1). México: Fondo de cultura económica.

Hirozawa, S. (1983). Galvanostaircae Polarization. Journal of the Electrochemical Society, 130, 1718-1721.

Uruchurtu Chavarin, J. (1991). Electrochemical Investigations of the Activation Mechanism of Aluminium. Corrosion, 47, 472-479.

\section{SOBRE LOS AUTORES}

\section{Luis Garita Arce}

Ingeniero Químico, investigador del Centro de Investigación en Corrosión del Instituto Costarricense de Electricidad.

Teléfono: 2220-5164

Correo: lgaritaar@ice.go.cr

\section{Lino Rivolta Carvallo}

Ingeniero de Materiales, con mención metalurgia. Jefe Unidad de Investigación y Desarrollo de CVG Aluminios Nacionales S.A. (CVG ALUNASA).

Teléfono: 2636-0000 ext. 220

Correo:linorivolta@gmail.com

\section{Mario Vega León}

Máster en Ingeniería Química, investigador del Centro de Investigación en Corrosión del Instituto Costarricense de Electricidad.

Teléfono: 2220-5164

Correo:mvegal@ice.go.cr 
